\title{
An Evolving-Graph-Based Finite State Machine Model for Protocol Conformance Testing in MANETs
}

\author{
Ting Shu, Mangmang Gu and Jinsong Xia \\ School of Information Science and Technology, Zhejiang SCI-Tech University, \\ Hangzhou 310018, China \\ shuting@zstu.edu.cn
}

\begin{abstract}
Dynamic network topology is one of the inherent features of Mobile Ad hoc Network (MANET). However, it may lead to unfeasible test sequences in the FSM-based active conformance testing, due to the topological distinction between testing sequences generation and its execution. In this paper, a formal model, named Evolving-Graph-based Finite State Machine (EGFSM), is proposed to help alleviate the impact of the dynamic network on conformance testing in MANETs. The evolving graph theory is first introduced to extend the Finite State Machine model to describe the dynamic behavior of the protocol with time-varying network topology. In the testing scenario with a predictable node movement pattern, test sequences adaptable to the dynamic topological changescan be generated on the basis of the EGFSM for the protocol under testing. A case study based on the AODV routing protocol is conducted, and promising experimental results show that it is a feasible way to test the protocols in MANETs using an EGFSM.
\end{abstract}

Keywords: MANET, dynamic topology, evolving graph (EG), conformance testing, test sequences

\section{Introduction}

In the last decades, advances in Mobile Ad hoc Network (MANET) [1] have brought about explosively increasing applications, ranging from military tactical networking to civilian instant networks deployment. Some protocol specifications, such as Ad hoc OnDemand Distance Vector Routing Protocol (AODV) [2], Dynamic Source Routing Protocol (DSR) [3] and Temporally Ordered Routing Algorithm (TORA), are established so as to drive the development and application of MANET technologies. After a protocol implementation is claimed to follow a specific standard, a protocol conformance testing should be carried out to validate whether the implementation under testing (IUT) is conformance to its specification. The mobility of nodes and unstable wireless communication quality make dynamic network topology be the inherent features of MANETs. The classical FSM-based active conformance testing methods require test sequences generation before the testing execution [4]. Due to topology change dynamically by nodes movement, the feasibility of generated test sequences becomes a central problem in MANETs. When we execute a set of conformance testing sequences, it is possible that the route corresponding to test sequences is no longer valid. As a consequence, the testing execution gives many INCONCLUSIVE verdicts [5]. Therefore, MANETs pose us some new challenges to model and test routing protocols in scenarios with time-varying network topology.

The main reasons for INCONCLUSIVE verdicts given in the traditional conformance testing execution procedure is that the network topology in test generation distinguishes with one in the test execution. In addition, the formal model for test sequences generation is generally constructed regarding to static test scenarios without the ability of adapting 
for the dynamic topology. To relieve the impact of node mobility on conformance testing execution, developing an adaptive finite state machine model is a feasible solution. Although the potentially node mobility leads to the frequently changing network topology, some MANETs have also the feature of a predicted node mobility pattern. These MANETs can be categorized as topology predicated dynamic networks (TPDNs), such as VANET [6]. In recent years, the evolving graph (EG) [12] has been presented as a formal description technique to describe the dynamic behavior of TPDNs, which was applied to optimize the route algorithm in a dynamic topology. Hence, EG theory also provides us a new ideal to construct an adaptive model for conformance testing in TPDNs.

The objective of this paper is to propose a novel method to construct an adaptive model for MANETs testing using the EG theory. In our proposed approach, a FSM model is extended to formally describe the behavior of dynamic network using EG, which is named EGFSM. The EGFSM can model a network protocol for conformance testing at a series of successive time intervals, regardless of the node movement. Therefore, EGFSMbased test sequence generation method can reduce the potential gap between the network topology of test generation and test execution. Consequently, the phenomenon of INCONCLUSIVE verdicts given in test execution can be alleviated effectively.

\section{Related Work}

As above mentioned, those inherent constraints pose significant challenges to formal testing for MANET protocols. In MANETs, the dynamic network topology by node movement is the greatest barrier to conformance testing using FSM [7-8].

In [9], the authors focus on the impacts of highly dynamic topology changes on the FSM modeling for MANET protocols under testing. Based on an equivalent division concept, they proposed a reduction method for the FSM model and the corresponding generated test cases. However, application of this method has some harsh restrictions, such as links between nodes are bidirectional. Unfortunately, it is difficult to meet these constraint conditions in a real MANET network. Then, Kojima [10] presents a FSMbased modeling method regarding the unidirectional links scenarios in MANETs. They redefines a given FSM model to reduce the number of the states and transitions using input and output sets. Similarly, this proposed method also concentrates on how to reduce the size of the given FSM and the number of its test cases. Little attention is given to the feasibility of test sequences generated from the reduced FSM model for a real testing execution.

With respect to the execution of the conformance test sequences in a real MANET with the dynamic topology, Maag [5] proposed an approach to improve the feasibility of the generated test sequences using a nodes self-similarity technique. Then, the network topology can be simplified into three representative nodes, just each time a packet of the test sequences is received or sent. Some inspired test results have been provided through test execution experiments regarding to the DSR routing protocol. However, when it is applied to a network with unpredictable nodes movement, the testing execution also gives many INCONCLUSIVE verdicts.

The reason for the challenge in testing execution for above existing methods is that testing models is established statically at the test generation procedure, which is unable to adapt to the dynamic topology changes in the execution. It is difficult to build a test model for a MANETs without a certain mobility pattern. But, for some predictable topology network, such as FSDNs [11], the evolving graph [12] can be utilized as a tool to abstract and describe the dynamic behavior. So it provides an opportunity for modeling and testing a dynamic topology network with predictable movement pattern.

\section{FSM-Based Protocol Testing in MANETs}


The FSM as a formal model has been widely applied in communication protocols testing [13]. A FSM can be represented as a six-tuple $\left(S, \mathrm{~s}_{0}, I, O, f_{s}, f_{o}\right) . S$ is a finite set of states. $\mathrm{s}_{0} \in S$ is the initial state. $I$ is the set of inputs; $O$ is the set of outputs; $f_{s}$ is the state transition function $f_{s}: S \times I \rightarrow S ; f_{o}$ is the output function $f_{o}: S \times I \rightarrow O$.

When the behavior of a network node in MANETs is formally represented using a FSM, its current snapshot of status is denoted as a state. The node keeps staying in a state, until it receives a data packet, called an input, to trigger a potential state transformation regarding the protocol specification. Commonly, the state transformation is modeled as a transition in the FSM, described as a three-tuple $\left(S_{i}, S_{j}, i / o\right)$, where the state $S_{i}$ changes into the state $S_{j}$, when the node receives an input packet $i$, and then forwards the output packet $o$ to the next hop node.

\section{Unfeasible Test Sequences Problem}

In a MANET network, the network connectivity among nodes often change due to the node movement. Hence, a forwarding node may have different neighbor nodes at different time intervals, as shown in Figure 1. The Figure 1 represents a forwarding nodeNf and its neighbor nodes at different four time intervals from T1 to T4. A source node, in an AODV routing protocol, disseminates a RREQ, when it needs a route to a specific destination node without an available route entry in the routing table. The forwarding node may receive RREQ packets at different time intervals from different source nodes. According to the AODV, the forwarding node will drop these packets and do nothing, if it receives the same RREQ packets from its neighbor nodes. The situation is also similar to other reactive routing protocols, such as DSR.

We choose the node Nf as the node under testing. In FSM-based protocol conformance test, there are increasing explosively number of states and transitions due to the dynamic topology. It poses a challenge on modeling the protocol under testing based on the FSM. In addition, there is a difference between the test sequences generation and test execution concerning the network topology due to the node movement. As an example, we generate the conformance testing sequences at the time interval $\mathrm{T} 1$, and then execute the generated test sequences in another time interval T3. For the node mobility, the input/output packets in the test sequence may lose their anticipated source and destination. Consequently, many inconclusive verdicts are obtained in the process of test execution, which leads to unfeasible conformance testing sequences.

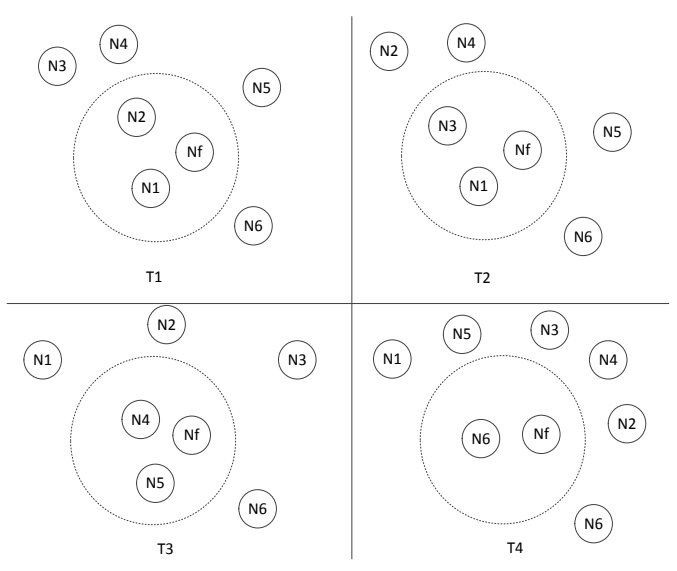

Figure 1. Networks Connectivity during different Time Intervals

\subsection{FSM-Based Testing Model for AODV}


States and transitions of a FSM for the network node in MANET are closely related to its neighbor nodes varied with the time. In AODV, one of the main reasons is that the result routes in a node is selected and maintained, according to the order of receiving a sequence of RREQ packets from its neighbor nodes [9]. Consequently, state enumeration with regard to different receiving order of RREQ packets form neighbor nodes will lead to a state explosion problem. Hence, it has been a hard work to apply the FSM model to describe the behavior of a node in MANET.

Let a network topology in AODV be divided into four time domains donated as T1, T2, T3 and T4, shown in Figure 1. It can be observed that Nf, the node under testing, has different neighbor nodes at different time intervals. Its neighbor nodes are denoted as N1, N2, N3 and so on. In Figure 1, suppose that Nf may receive and then forward the RREQ packet in the time domain T1, but not receive RREP packet from its any neighbor node before the end of the time domain T4. Hence, Nfwill receive different RREQ packets from different neighbor nodes at different time intervals. So, in the FSM model of the forwarding node $\mathrm{Nf}$, the set of inputs $I_{n}$ can be represented by expression (1), where $r e q_{i}$ is the RREQ from the node $\mathrm{N}_{i}$ and $n$ represents the number of its neighbor nodes. $S_{0}$ is the initial state. when $\mathrm{Nf}$ receivesand forwards the packet $r e q_{1}$, the state will change into $S_{1} . S_{12}$ means that the state $S_{1}$ receives $r e q_{2}$, as shown in the expression (2) and expression (3).

$$
\begin{aligned}
& I_{n}=\left\{r e q_{1}, r e q_{2}, r e q_{3}, \ldots, r e q_{n}\right\} \\
& \left(S_{0}, S_{1}, r e q_{1} / r e q_{1}\right) \\
& \left(S_{1}, S_{12}, r e q_{2} / r e q_{2}\right)
\end{aligned}
$$

For convenience, we define the following symbols and definitions before the description of our method in detail. $T_{i}$ represents a time domain, $i \in[1,2,3,4, \ldots, n] . S^{(i)}$ representsthe set of states existing in the time domain $T_{i}$ excluding the initial state, $i \in[1,2,3,4, \ldots, n] . S^{\prime(i)}$ represents the set of all the states in a time domain , include the initial state $\mathrm{S}_{0}$. The reqi is a set of RREQ packets receiving from neighbor nodes in the time domain $T_{i}, i \in[1,2,3,4, \ldots, n]$. The rep ${ }^{i}$ represents a set of RREP packets that the forwarding node receives from the destination node regarding a RREQ initiating in the time domain $T_{i}, i \in[1,2,3,4, \ldots, n]$.

\section{EGFSM Model}

In this section, we present a new model for conformance testing, named Evolving Graph-based Finite State Machine (EGFSM), to formally describe the routing protocol in MANETS.

\subsection{Evolving Graph Theory}

The evolving graph [14] is proposed to formally represent dynamic networks, through the combination of a suite of sub-graphs indexed by the time interval number according to the network connectivity. As an example, an evolving graph is built based on the network connectivity for the four time intervals of the dynamic network in Figure 1, as depicted in Figure2. As we can observe, it is only the partial evolving graph concentrating on the network connectivity between $\mathrm{Nf}$ and its neighbor nodes. In an evolving graph, a transition is labeled with a sequence of time index number in a non-decreasing order. Note that the transition, between the node Nf and N1 in Figure 2, represents there exists a network connectivity at the time domain $\mathrm{T} 1$ and $\mathrm{T} 2$. 


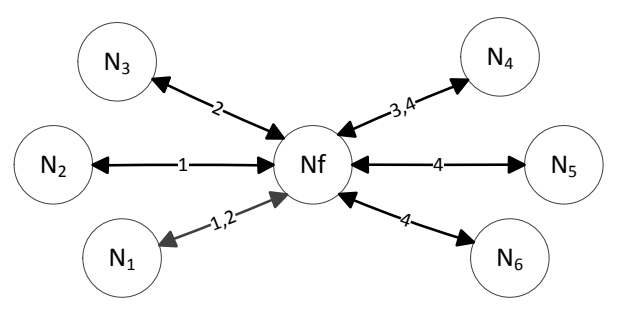

Figure 2. Evolving Graph Example

Obviously, the evolving graph provides an feasible means to describe the dynamic topology with a known movement pattern. However, it cannot be directly utilized to build a state machine model for MANET testing. This paper try to merge the evolving graph theory into a FSM model to support for description of the dynamic behavior.

\subsection{Evolution of a FSM}

Based on above discussion and definitions, the FSM of the node $\mathrm{Nf}$ can be evolved into the four snapshots, as depicted in Figure 3, according to the four time domains of Figure 1, respectively. For simplicity, this paper only considers the situation that a forwarding node can receive the RREP from neighbor nodes without timeout. For convenience of discussion, we minimize the interval time in Figure 3 to one second, which is equal to HELLO_INTERVAL according to the RFC3561. Hence, the forwarding node Nf have enough time to discover its all neighbor nodes during a time domain. The circle represents the state and the curve line with an arrow represents a transition. The state of the FSM model for Nf is determined by the order of received RREQ packets. Specially, the initial state is $S_{0}$. Nf may receive many different RREP packets. When receiving a RREP packet at anytime, the state will return to $S_{0}$. Hence, the set of state regarding the four time intervals can be represented as following: $S^{(1)}=\left\{S_{1}^{(1)}, S_{2}^{(1)}, S_{\text {all }}^{(1)}\right\}$, $S^{(2)}=\left\{S^{(1)}, S_{1}^{(2)}, S_{3}^{(2)}, S_{\text {all }}^{(2)}\right\} \quad, \quad S^{(3)}=\left\{S^{(2)}, S_{\text {all }}^{(3)}\right\}$ $S^{(4)}=\left\{S^{(3)}, S_{4}^{(4)}, S_{5}^{(4)}, S_{6}^{(4)}, S_{45}^{(4)}, S_{46}^{(4)}, S_{56}^{(4)}, S_{\text {all }}^{(4)}\right\}$.

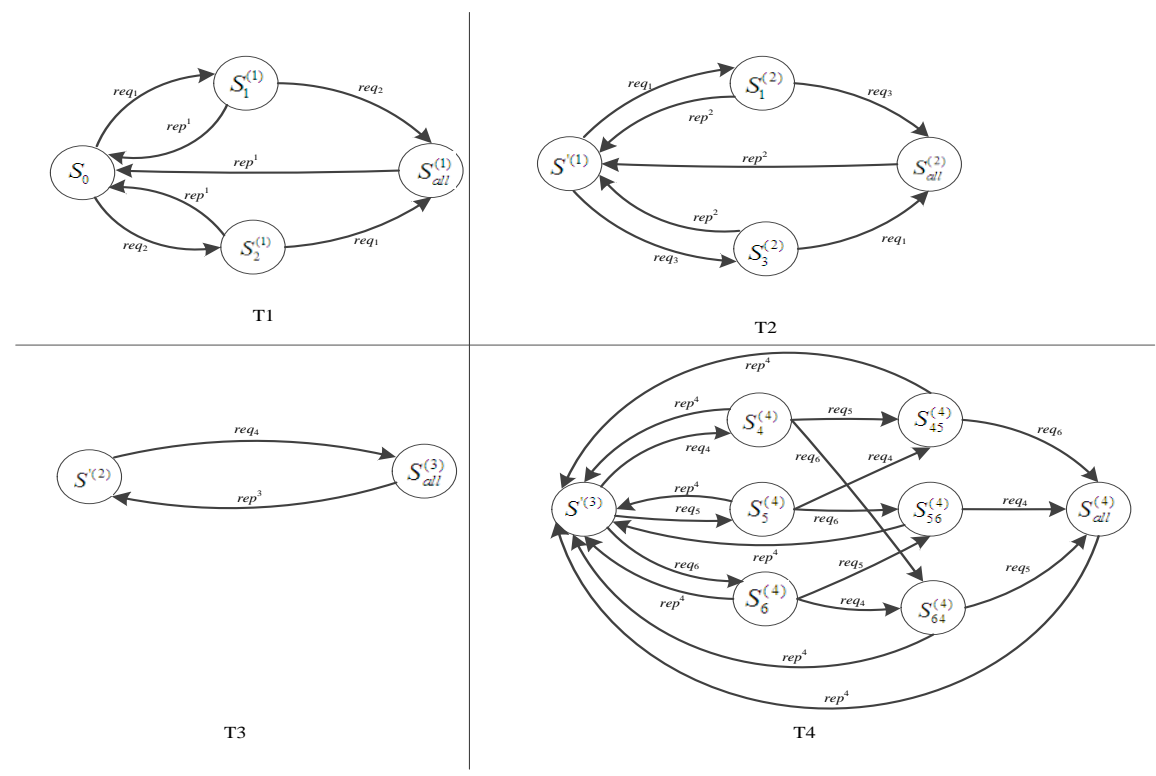

Figure 3. FSMs at Four Time Intervals 
With the increasing number of neighbor nodes, the states and transitions of the FSM will explosively increase. In AODV, when a node receives a RREQ from its neighbor nodes, it will check whether it has received a RREQ with the same Originator IP Address and RREQ ID or not. If so, the new arrived RREQ will be discarded without any further processing. In order to compress the size of the FSM for conformance testing, some states and transitions can be treated equivalently as a set of state and transition, respectively. As an example, $S_{i j}$ and $S_{j i}$ are equivalent, if $r e q_{i}$ and $r e q_{j}$ have the same information from $\mathrm{N}_{i}$ and $\mathrm{N}_{j}$.So we also can regard $r e q_{i}$ and $r e q_{j}$ as the equivalent inputs, denoted as a set. Consequently,we can reduce the number of states and transitions using the equivalent description method for inputs and states.

Based on the equivalent description method, the four FSM models in Figure 3 can be redefined into the following new four models, as shown in Figure 4, respectively. In Figure 4, we can consider the inputs at different time intervals as a input set, such as req ${ }^{i}$ and $r e p^{i}$. The neighbor nodes may receive a series of RREQ packets from different source nodes.

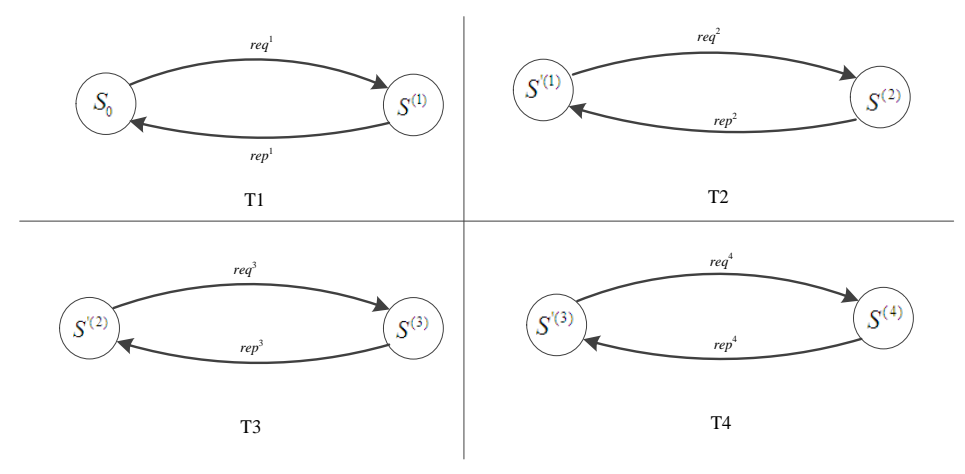

Figure 4. Evolution of FSMs

\subsection{The EGFSM Model}

An Evolving Graph-based Finite State Machine model extends the FSM model regarding the description capability for the dynamic behavior of a protocol. The EGFSM is a six-tuple $M=\left(S_{0}, S, I, O, T I, T\right) . S_{0}$ is the initial state. $S=\left\{S_{0}, S_{(1)}, S_{(2)}, \ldots, S_{(n)}\right\}$ is the finite set of state during a series of time intervals and $n$ equal to the time interval count. $I=\left\{I^{1}, I^{2}, \ldots \ldots, I^{n}\right\}$ is the finite set of inputs, where $I^{n}$ represents the specific input set and $n$ is also the number of the time interval. Similarly, $O=\left\{O^{1}, O^{2}, \ldots, O^{n}\right\}$ is the finite set of outputs. $T I$ is the set of the time interval index, $T I=\{1,2, \ldots \ldots, n\}$ and $n \in \mathrm{N}$. $T$ represents the transition set. A transition $t_{i} \in T$ is a five-tuple $\left(\mathrm{S}_{(i)}, \mathrm{S}_{(j)}, I^{i}, O^{i}, t i\right)$, where $\mathrm{S}_{(i)}$ and $\mathrm{S}_{(j)}$ is the head state and tail state, respectively. When receiving an input $I^{i}$ at $\mathrm{S}_{(i)}$ during the time intervals $t i \subseteq T I, M$ gives an output $O^{i}$ and the state transfers into $\mathrm{S}_{(j)}$.

As an example, the four FSM models in the Figure 4 can be redefined into a single EGFSM model, as depicted in Figure 5. The transition $t_{1}$ represents that the IUT receives the RREQ packet $r e q^{1}$ and forwards it to the neighbor nodes in the time domain T1. Because the EGFSM in the Figure 5 describes the behavior of IUT in the testing scenario, from forwarding a RREQ to receive a RREP, it will return to initial state if it receives a rep. Therefore, when the IUT receives a RREP packet $r e p^{3}$ in the T3, its state will transfer from $S^{(3)}$ to $\mathrm{S}_{0}$. 


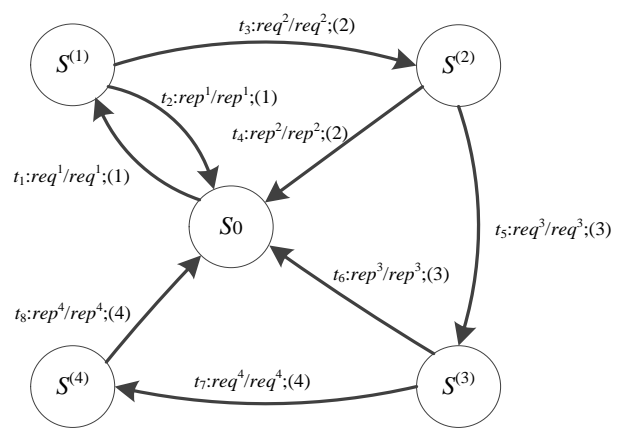

Figure 5. An EGFSM Model

\subsection{EGFSM-Based Test Sequence Generation}

Conformance testing sequences can be generated based on the built EGFSM regarding the scheduled test scenarios. Set $\mathrm{S}_{0}$ as the initial State. According to the EGFSM shown in the Figure 5, the four test scenarios can be classified by the time when the IUT receives a RREP packet.

Take the time domain T1 as an example. The IUT receives RREQ packets from its neighbor nodes and forwards themat the initial state $S_{0}$. If it doesn't receive a RREQ packet in the T1, the state transfers into $S^{(1)}$. Otherwise, the state will return into the initial state $S_{0}$. Similarly, we can derive the other three testing scenarios. We utilize the Message Sequence Chart (MSC) to depict four testing scenarios, as shown in Figure 6. As a result, four test sequences can be generated according to these testing scenarios, as shown in the Table 1.

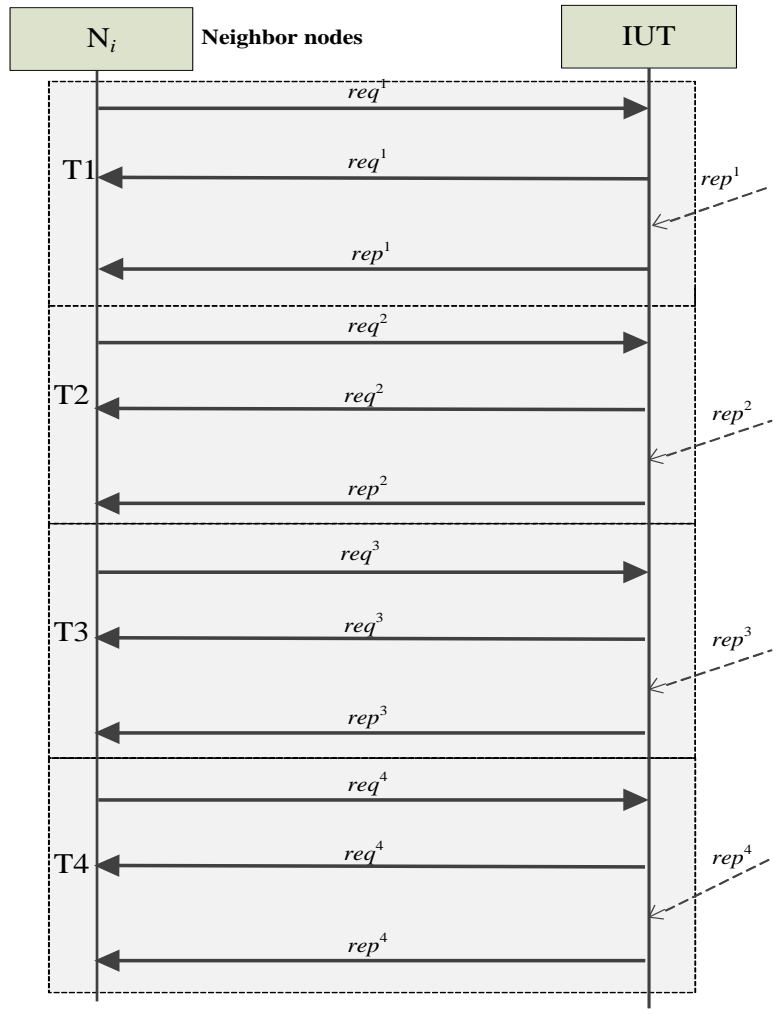

Figure 6. Simplified MSC for Four Testing Scenarios 
Table 1. Generated Test Sequences

\begin{tabular}{c|l}
\hline ID & \multicolumn{1}{|c}{ Test sequences } \\
\hline 1 & $t_{1}, t_{2}$ \\
\hline 2 & $t_{1}, t_{3}, t_{4}$ \\
\hline 3 & $t_{1}, t_{3}, t_{5}, t_{6}$ \\
\hline 4 & $t_{1}, t_{3}, t_{5}, t_{7}, t_{8}$ \\
\hline
\end{tabular}

\section{A Case Study}

In this section, we designs a test execution experiment to validate the effectiveness of the proposed model and its generated test sequences, regarding the protocol conformance testing for AODV. Figure 7 describes an AODV network topology with predictable dynamic patterns at different four time intervals. The dotted circle denote the communication rang of the node under testing, named $\mathrm{N}_{10}$. In any time domain, $\mathrm{N}_{10}$ may receive RREQ and RREP packets from its neighbor nodes in the dotted circle. In time domain $\mathrm{T} 1, \mathrm{~N}_{10}$ has three neighbor nodes, $\mathrm{N}_{1}, \mathrm{~N}_{2}$ and $\mathrm{N}_{3}$. In the next three time domains, neighbor nodes of $\mathrm{N}_{10}$ varied due to their movement. Finally, $\mathrm{N}_{10}$ 's neighbor nodes become $\mathrm{N}_{1}, \mathrm{~N}_{3}, \mathrm{~N}_{7}$ and $\mathrm{N}_{9}$.

In this case study, suppose that the forwarding node $\mathrm{N}_{10}$ only accepts the RREQ packet in T4. That is, $\mathrm{N}_{10}$ will receive RREQ packets from its vary neighbor nodes without any RREP packet. Under this situation, the procedure of test execution is detailed in the following steps in Table2.

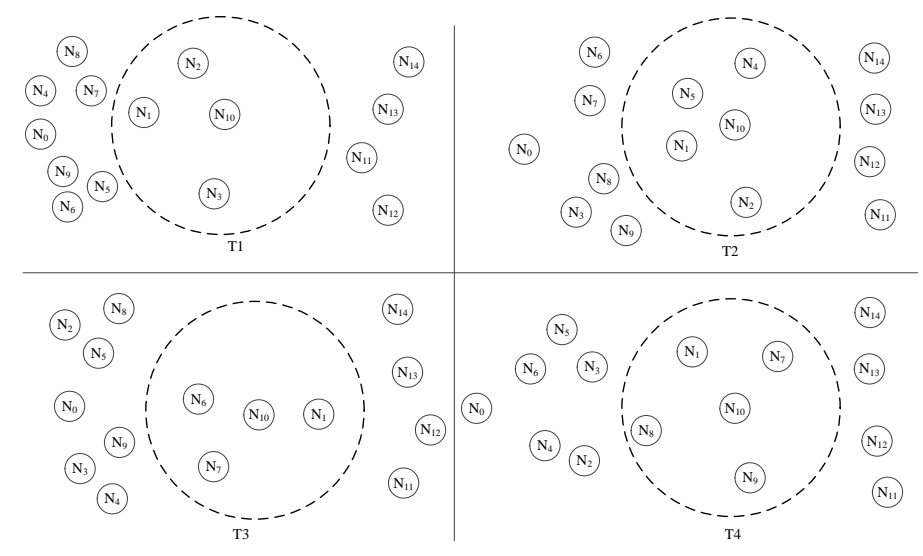

Figure 7. Testing Scenarios of AODV

According to the test execution procedure depicted in Table 2, we can observe that the executed test sequence is the 3rd test sequence in table 1. It is obviously see that the generated test sequences from EGFSM model can adaptive for the specific testing execution scenario. Consequently, the side-effect of the topology distinction between test generation and test execution can be reduced effectively.

Table 2. Procedure of Test Execution

Step 1. In T1, $\mathrm{N}_{10}$ receives and forwards $r e q^{1}$. The transition $t_{1}$ is executed.

Step 2. $\mathrm{N}_{10}$ receives $r e q^{2}$ in time domain T2. Hence, the executed sequence becomes $t_{1}, t_{3}$.

Step 3. In time domain T3, $N_{10}$ receives and forwards $r e q^{3}$. The executed sequenceis $t_{1}, t_{3}, t_{5}$.

Step 4. Finally, $\mathrm{N}_{10}$ receives $r e p^{3}$ in time domain T4.The final executed test sequence is $t_{1}, t_{3}, t_{5}, t_{6}$. 


\section{Conclusion}

In this paper, a formal model named EGFSM is proposed to describe the behaviors of protocols in MANET for conformance testing. The proposed method introduces the evolving graph theory to extend the FSM model to enhance the description capacity for the dynamic behavior. Assume that the topology of the protocol under testing is predictable. test sequences generated from EGFSM can adaptive for the specific network topology in the test execution. Hence, INCONCLUSIVE verdicts can be effectively reduced in protocol conformance testing under the dynamic testing scenario. A case study shows that it is a promising try to apply the proposed method to AODV routing protocol. In our future work, we will focus on the automatic test sequence generation based on the EGFSM.

\section{Acknowledgments}

We would like to appreciate the anonymous reviewers for their constructive comments and suggestions to improve the quality of this paper. This work is partially supported by the National Natural Science Foundation of China (No.61101111).

\section{References}

[1] M. Conti and S. Giordano, "Mobile ad hoc networking: milestones, challenges, and new research directions", IEEE Communications Magazine, vol. 52, no. 1, (2014), pp. 85-96.

[2] C. Perkins, E. Belding-Royer and S. Das, "RFC 3561-ad hoc on-demand distance vector (AODV) routing", Internet RFCs, (2003).

[3] D. Johnson, D. Maltz and Y.-C. Hu, "The dynamic source routing protocol for Mobile Ad Hoc Networks (DSR) - Experimental RFC", Internet RFCs, (2004).

[4] R. Dorofeeva, K. El-Fakih, S. Maag, A. R. Cavalli and N. Yevtushenko, "FSM-based conformance testing methods: A survey annotated with experimental evaluation", Information and Software Technology, vol. 52, no. 12, (2010), pp. 1286-1297.

[5] S. Maag, C. Grepet and A. Cavalli, "A formal validation methodology for MANET routing protocols based on nodes' self-similarity", Computer Communications, vol. 31, no. 4, (2008), pp. 827-841.

[6] M. H. Eiza and Q. Ni, "An evolving graph-based reliable routing scheme for VANETs", IEEE Transactions on Vehicular Technology, vol. 62, no. 4, (2013), pp. 1493-1504.

[7] A. C. Viana, S. Maag and F. Zaidi, "One step forward: Linking wireless self-organizing network validation techniques with formal testing approaches", ACM Computing Surveys, vol. 43, no. 2, (2011), pp. 7:1-7:36.

[8] K. EI-Fakih, N. Yevtushenko and G.V. Bochmann, "FSM-based incremental conformance testing methods", IEEE Transactions on Software Engineering, vol. 30, no. 7, (2004), pp. 425-436.

[9] H. Kojima, J. Takahashi and Y. KAKUDA, "An equivalent division method for reducing test cases in state transition testing of manet protocols", IEICE transactions on communications, vol. 92, no. 3, (2009), p. 794-806.

[10] H. Kojima, T. Ohta and Y. Kakuda, "A Transition Reduction Method for FSM of MANET Routing Protocol with Blacklist", (2011), pp. 611-616.

[11] J. Monteiro, A. Goldman and A. Ferreira, "Performance evaluation of dynamic networks using an evolving graph combinatorial model", IEEE International Conference on Networking and Communications in Wireless and Mobile Computing (WiMob'2006), (2006), pp. 173-180.

[12] G. Mao and B. Anderson, "Graph theoretic models and tools for the analysis of dynamic wireless multihop networks", IEEE Wireless Communications and Networking Conference, (2009), pp. 1-6.

[13] A. Petrenko and N. Yevtushenko, "Testing from partial deterministic FSM specifications", IEEE Transactions on Computers, vol. 54, no. 9, (2005), pp. 1154-1165.

[14] A. Ferreira, "Building a reference combinatorial model for MANETs", IEEE Network, vol. 18, no. 5, (2004), pp. 24-29. 


\section{Authors}

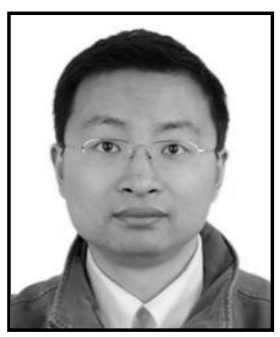

Ting Shu, received the Ph.D. degree in Computer Science from Zhejiang University in 2010. He is now an associate professor with the School of Information Science and Technology, Zhejiang Sci;Tech University, 310018, Hangzhou, China. His current research interests include software testing and network protocol testing.

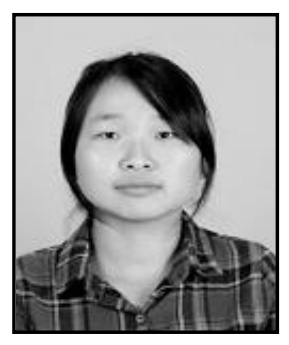

Mangmang Gu, received her Bachelor of Engineering in Computer Science and Technology from Anhui university of science and technology, CHINA in 2009. Now she works in the field of master's studies. She is mainly researching on Protocol conformance testing and the Computer network.

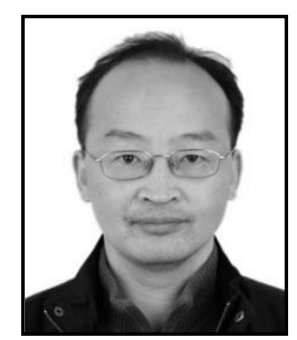

Jinsong Xia, is a $\mathrm{PhD}$. candidate in Control Theory and Control Engineering at School of Mechanical Engineering\& Automation and a lecturer at the School of Information Science and Technology, Zhejiang Sci.-Tech University,310018, Hangzhou, China. He received his M.S. in computer science from Zhejiang University in 2006. His current research interests are mainly in software testing. 